\title{
Assessment of the CPL-0015 isolate as a vaccine strain for the control of canine parvovirus in Cuba
}

\section{INTRODUCTION}

Canine parvovirus (CPV) is the cause of serious enteric infections in Canis familiaris, resulting in acute clinical progression with high morbidity and mortality regardless of age, race, or gender (Aponte et al 2020, Dong et al 2020). However, several authors consider that certain factors such as younger age, nonconfinement, and lack of vaccination play a key role in its spread, clinical course, and fatal outcome (Zhuang et al 2019, Qi et al 2020). CPV is characterised by its genetic diversity and alarming ability to cross the interspecies barrier, enabling it to adapt to multiple hosts, both domestic and wild (Miranda and Thompson 2016, Li et al 2017, Zhou et al 2017, Voorhees et al 2019).

The immunisation of domestic dogs with modified live vaccines containing the CPV-2 strain is an effective method of controlling and preventing the clinical development of the disease (Domínguez et al 2014, Mukthar et al 2021). However, in recent years the use of these formulations has become controversial due to their failure in protecting vaccinated animals (Decaro et al 2020, Ying et al 2020). Although the cause of these events is multifactorial (Altman et al 2017), much attention is being paid to the possible inability of the CPV-2 vaccine strain to induce protection

Received: 27.07.2021.

Accepted: 25.11.2021.

${ }^{a}$ Empresa Productora de Vacunas Virales y Bacterianas, Grupo Empresarial LABIOFAM, La Habana, Cuba.

bDirección de Investigación y Desarrollo, Grupo Empresarial LABIOFAM, La Habana, Cuba.

${ }^{\mathrm{C}}$ Grupo de Investigación en Ciencia Animal, Universidad Cooperativa de Colombia, Bucaramanga, Colombia.

*Corresponding author: DL Cala Delgado; Carrera $33 \mathrm{~N}^{\circ} .30 \mathrm{~A}-05$ (4.162,49 km) 68000, Bucaramanga, Colombia; daniel.cala@ campusucc.edu.co against the new genetic variants CPV-2a, CPV-2b, and CPV-2c (Ying et al 2020). Such suspicions are supported by the antigenic variations detected in emerging lineages associated with several mutations located in the VP2 structural protein, an important antigenic determinant present in the viral capsid ( $\mathrm{Li}$ et al 2017, Sebastian et al 2019, Ying et al 2020).

As a result, among other aspects, there is an urgent need to evaluate the immunological consequences of the genetic diversity of CPV, the genotypes prevailing in different countries, the antigenic properties of each one, and their possible vaccine potentialities (Zhou et al 2017, Sebastian et al 2019, Hao et al 2020). The antigenic variability observed at the global level does not seem to be present in Cuba. Molecular studies (results not presented) carried out after those published by Fresneda et al (2015) from diseased animals, demonstrated the circulation of the CPV-2 strain only. The existence in our archipelago of original pathogenic strains that persist over time is not exclusive to canine parvovirus, it was also reported for Pasteurella multocida subsp multocida biovar A:1 in rabbits (Lugo et al 2019, Domínguez et al 2021).

Recent Cuban epidemiological studies revealed that no racial dogs with free access to the street, the consumption of inadequate food including raw meat, and not vaccination are factors that increase the chances of becoming ill from CPV (Pino et al 2019, Peña et al 2020). In this context, having its own vaccine will make it possible not to depend on international suppliers of vaccines to control the disease. Besides, the possibility of achieving technological sovereignty would reduce the prices of the biological product and promote its commercialisation in the foreign market. This perspective led us to isolate, attenuate in cell culture, and characterise circulating strains native to Cuba (Fresneda et al 2015). Accordingly, a vaccine formulation 
was developed with our own technology, adapted to the Cuban epidemiological reality, independently of external strains. Consequently, the present study assessed the safety and protective efficacy of the Cuban isolate, CPL0015, of canine parvovirus type 2 as a vaccine strain in Beagle dogs without specific maternal antibodies against parvovirus canine.

\section{MATERIAL AND METHODS}

EXPERIMENTAL ANIMALS

This experiment followed national (GOC-2021332-EX25) and institutional guidelines for the care and use of animals. All experimental procedures were approved by the Committee of Ethical Review at Grupo Empresarial LABIOFAM (protocol approval number: 06/20; experimental period: October 2020). The selection of the biomodel, age and number of individuals per experimental group took into account several existing international references on clinical evaluations of classic and modern vaccines against canine parvovirus (Langeveld et al 2001, Siedek et al 2011, Hernández et al 2015). The study included a total of 23 healthy Beagle dogs of both sexes, aged 84 days (12 weeks), with no history of vaccination, testing negative for maternal antibodies against canine parvovirus (absorbance $<0.18$ ) and free of bacterial (Salmomella spp., Escherichia coli, Leptospira spp., Borrelia burgdorferi, Ehrlichia canis and Mycoplasma haemocanis), viral (Canine parvovirus, Canine hepatitis virus, Canine distemper virus and Canine rabies) and parasite (Nematode spp., Cestode spp., Babessia spp. and Ectoparasite) specific diseases for the species. The animals were placed in separate cages and were provided with adequate food based on the species and age (three times a day) and free access to water. After the adaptation period (seven days), the animals were randomly distributed (15 for the safety study and 8 for the efficacy study).

\section{IMMUNOGENS}

Inoculum. The candidate vaccine strain CPL-0015 (CPV-2) was isolated in 1991, attenuated by 53 passes in MadinDarby Canine Kidney cells (MDCK) and then stabilised through 27 passes in Crandell-Rees Feline Kidney Cell (CRFK) (Fresneda et al 2015). A vial of lyophilised CPL0015 isolate (Batch 1203010) with a titer of $10^{6.0}$ tissue culture infectious dose $(\text { TCID })_{50} / \mathrm{mL}$ was reconstituted with $1 \mathrm{~mL}$ of minimum essential medium. This volume was inoculated into the CRFK line supplemented by $2 \%$ fetal bovine serum. After incubating for seven days at $37^{\circ} \mathrm{C}$ and observing the cytopathic effect in more than $80 \%$ of the culture, we proceeded to collect, freeze-dry, and preserve it at $-70^{\circ} \mathrm{C}$. Before use, one of the obtained virals was diluted with water for injection to obtain a titer of $10^{6.0} \mathrm{TCID}_{50}$ in a total volume of $1 \mathrm{~mL}$ (figure 1 ).

Challenge strain. Over the base of previous international experiences (Cunegündes et al 2008, Oliveira et al 2010, De Cramer et al 2011, Puentes et al 2012), the worldwide virulent Cornell-780916 strain (type 2) from the collection ATCC with the number ATCC ${ }^{\circledR}$ VR- $2006^{\mathrm{TM}}$ was selected. Before its use, it was titled in the MDCK cellular line with a value of $10^{6.5}$ and then diluted with minimum essential media to obtain a titer of $10^{5.0} \mathrm{TCID}_{50}$ in a total volume of $1 \mathrm{~mL} /$ animal (figure 1 ).

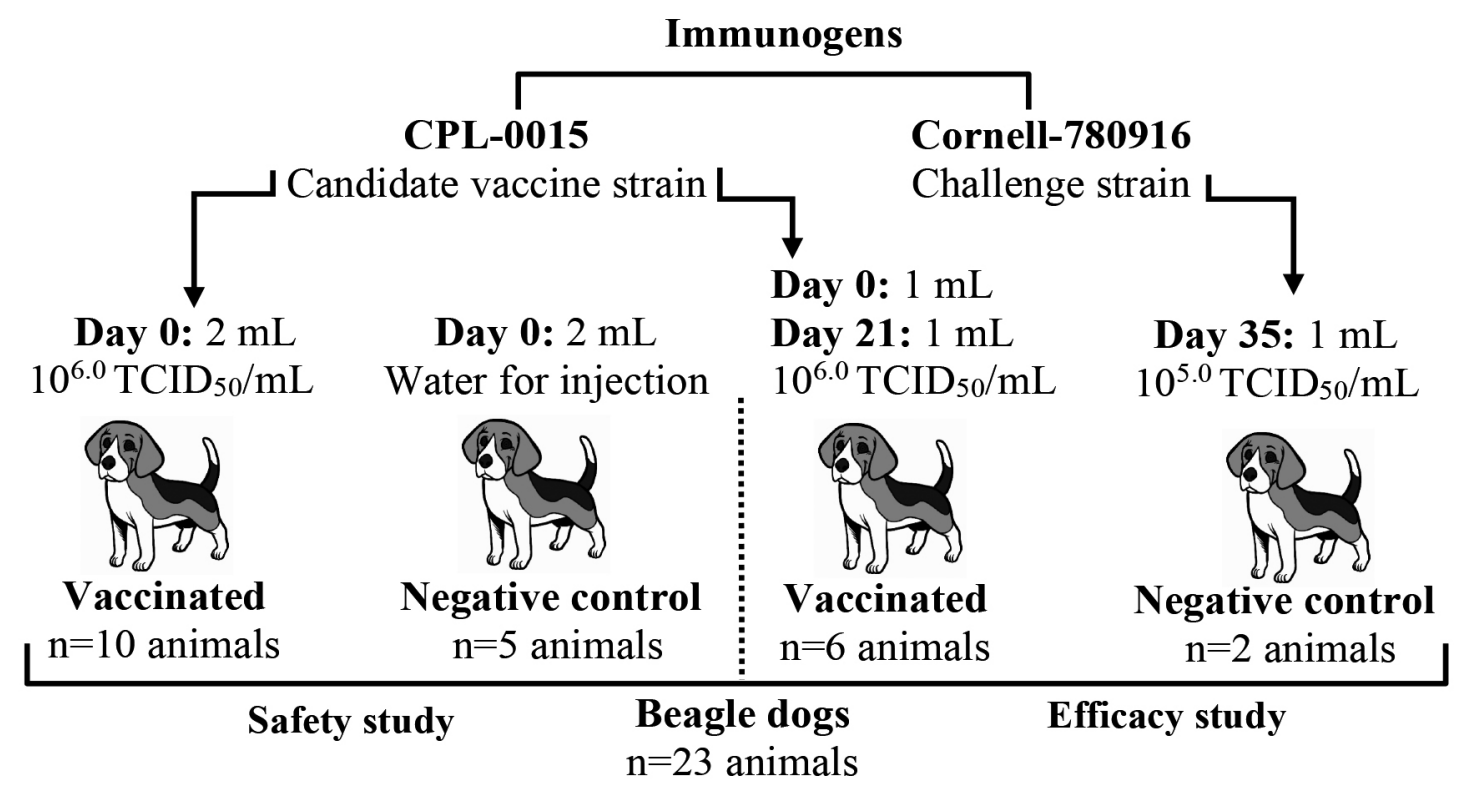

Figure 1. General aspects of the designed studies to evaluate the efficacy and safety of the CPL-0015 strain. 


\section{SAFETY STUDY}

Two experimental groups were established (figure 1). One group was inoculated with CPL-0015 at a titer of $10^{6.0}$ $\mathrm{TCID}_{50} / \mathrm{mL}(\mathrm{n}=10)$, whereas the negative control group received water for injection $(n=5)$. All animals received double doses ( $2 \mathrm{~mL}$ ) subcutaneously in the dorsal area of the neck and were manipulated by the same researcher. The injection site was observed for 14 consecutive days to look for local alterations and the animals were monitored for general clinical manifestations or changes in the rectal temperature. Furthermore, white blood cell counts, food consumption, and body weight were assessed on alternate days.

A clinical assessment system was followed based on possible and typical disease alterations. Scores ranged from 1 to 9 depending on the severity of the clinical alterations (table 1). Euthanasia was performed using a sodium pentobarbital (Pentovet, $150 \mathrm{mg} / \mathrm{kg}$, intraperitoneal) overdose when the score was greater than 7 .

\section{EFFICACY STUDY}

The animals used in this study were divided into two experimental groups (figure 1). One group $(n=6)$ was inoculated with two subcutaneous doses (1 mL each) of CPL-0015 (10 $\left.0^{6.0} \mathrm{TCID}_{50}\right)$ with an interval of 21 days between doses (days 0 and 21), whereas the negative control group $(n=2)$ received water for injection in the same volume, frequency, and route of administration as those of the inoculated group. Fourteen days after the second injection (experimental day 35), all animals were challenged orally with the virulent Cornell strain. Clinical follow-up of all animals was performed from day 0 to experimental day 56 using the abovementioned clinical assessment system. The first 35 days, were dedicated to observe the possible adverse reactions of the CPL-0015 initial dose and the recollection, using the same written indicators previously described.

Table 1. Clinical assessment system used in the safety study.

\begin{tabular}{cl}
\hline Clinical score & \multicolumn{1}{c}{ Clinicopathological alterations } \\
\hline 1 & No symptoms \\
2 & Pain at the injection site \\
3 & Redness at the injection site \\
4 & Unusual skin manifestations at the injection \\
& site (erythema, alopecia, etc.) \\
5 & Fever + decreased willingness to eat and drink \\
7 & Mucoid or hemorrhagic diarrhea \\
8 & Vomiting + mucoid or hemorrhagic diarrhea \\
9 & Anorexia + weakness + prostration \\
\hline
\end{tabular}

HAEMATOLOGY AND SEROLOGY

Blood samples for assessing the white blood cell counts of the animals in the safety study were collected on experimental days $0,2,4,6,8,10,12$, and 14 . In all cases, $1 \mathrm{~mL}$ of blood was collected from the lateral saphenous vein into tubes containing an anticoagulant. Additionally, the evaluation of anti-CPV $\operatorname{IgG}$ antibody titers was performed on days 0,35 , and 56 using $2 \mathrm{~mL}$ of heat-inactivated serum $\left(56^{\circ} \mathrm{C}\right.$ for 30 minutes $)$. A solid phase, quantitative, indirect enzyme-linked immunosorbent assay system was used (CENPALAB, Cuba). The reading of the samples was taken at $492 \mathrm{~nm}$, and the cut-off value was 0.18 according to the manufacturer's instructions.

\section{STATISTICAL ANALYSIS}

For the analysis of immune response variables, data from the inoculated and control groups were compared using the Wilcoxon-Mann-Whitney test. A value of $P<0.05$ was considered statistically significant. The statistical program used for the analyses was SPSS 12.0 (SPSS Inc, Chicago IL, USA).

\section{RESULTS AND DISCUSSION}

Vaccination and use of live attenuated vaccines manufactured using classic production technologies continue to be essential tools for the control of canine parvoviruses worldwide (Puentes 2012, Domínguez et al 2014, Mukthar et al 2021). Therefore, controlled clinical studies using autochthonous circulating strains adapted to cell culture are important in order to produce immunoprophylactic formulations adapted to current epidemiological situations.

\section{SAFETY STUDY}

Clinical observation for 14 consecutive days after the inoculation of an overdose of the Cuban isolate, CPL-0015, demonstrated the absence of undesirable local or systemic side effects. Analysis of the white blood cell count, rectal temperature, food consumption, and body weight showed that the inoculation of CPL-0015 did not have a negative impact on any animal under these conditions (data not shown). The negative control group, on the other hand, showed similar results and clinical scores (level 1) as those of the inoculated group.

The use of clinical indicators to detect the occurrence of negative events associated with immunoprophylactic formulations (Day 2008) and the evaluation of vaccine antigens (Moore and Hogen 2010) is well-known. In our case, the lack of adverse consequences in animals inoculated with a CPL-0015 overdose supports its suitability for use in puppies. This characteristic of CPL-0015 will be greatly beneficial if used in future formulations because although it is a living virus belonging to the family Parvoviridae, it 
shows a high affinity for growing cells having a high rate of mitotic division (Díaz et al 2008).

\section{EFFICACY STUDY}

Figure 2 summarises the antibody response of the Beagle dogs from both the experimental and control groups at different time intervals. Prior to inoculation (day 0), anti-CPV antibodies were undetectable (values below 0.18) with the technique used, confirming the absence of prior immunological stimulation. On day 35 , at the time of the challenge, all animals inoculated with CPL-0015 (days 0 and 21) showed a significant percent increase (490\%) in their antibody levels compared to the control group animals who continued to be seronegative. On experimental day 56 (21 days after the challenge), although in general terms significant progressive increases in IgG levels were found in both groups $(P>0.05)$, these were not homogeneous. Animals inoculated with CPL-0015 showed the greatest and most significant percent increases in their antibody levels not only with respect to days 0 and 35 (1,460\% and $297 \%$, respectively) but also with respect to the control group (129\%) in the same period.

Furthermore, figure 3 shows the results in terms of clinical score for the experimental and control groups before and after the challenge. In animals inoculated with CPL-0015, there was an absence of adverse local or systemic reactions from days 0 to 14 after the second injection (experimental days 35) and signs of disease after the challenge. In contrast, unvaccinated animals challenged with the virulent Cornell-780916 strain began to show progressive clinical signs typical of CPV from experimental day 39 (day 4 after the challenge). Control animals shared the same score until day 13 after the challenge, when

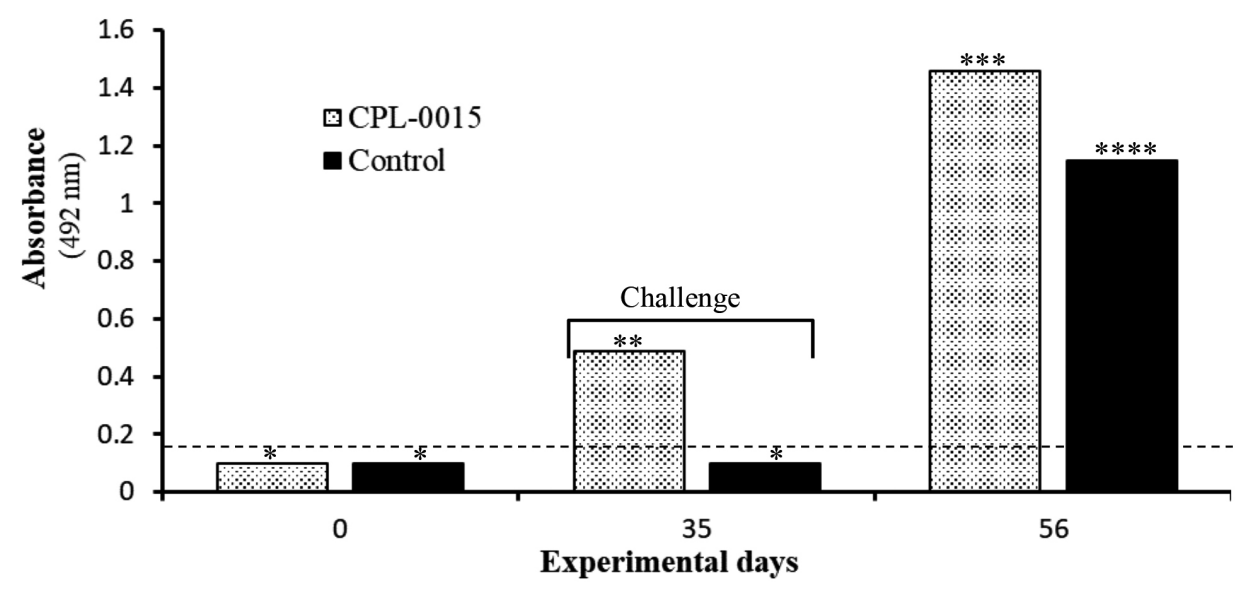

Figure 2. Immune response of Beagle dogs inoculated with strain CPL-0015 and controls.

Note: Different number of symbols indicate significant differences $(P<0.05)$ based on the Wilcoxon-Mann-Whitney test.

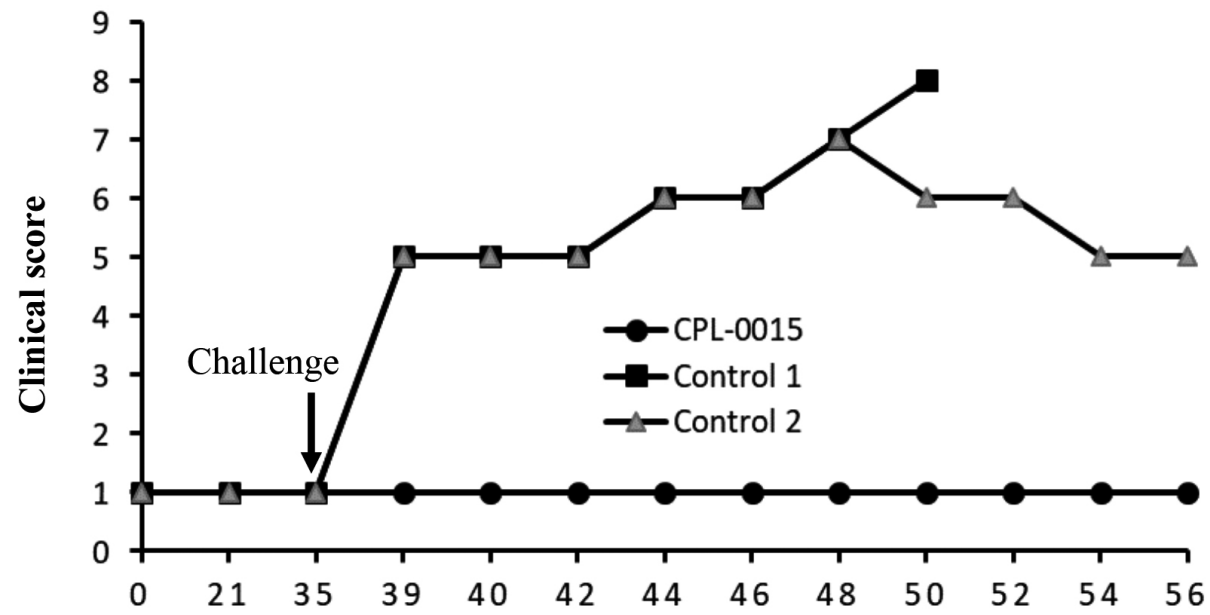

Experimental days

Figure 3. Clinical evaluation of Beagle dogs immunised with strain CPL-0015 (and of negative controls) challenged with the virulent Cornell-780916 strain.

Note: Group vaccinated with CPL 0015 is composed by 6 animals and control group by 2 animals. 
one of them progressed towards anorexia, weakness, and prostration, and had to be euthanised.

The remarkable biological differences in terms of seroconversion and survival without clinical signs of disease after the challenge observed between inoculated and control animals demonstrate the ability of the Cuban isolate to induce a powerful protective humoral response. These results are extremely important since they show that the CPL-0015 isolate retained its immunogenic capacity during the process of adaptation to cell culture and was able to induce anti-CPV antibodies in sufficient quantities to neutralise the pathogenic strain (Puentes 2012).

When comparing this result with others reported in the international scientific literature, it can be concluded that the benefits of the CPL-0015 isolate in terms of protection are similar to those obtained with the 17/80 ISS (Pratelli et al 2001), Cornell-780916-115 (De Cramer et al 2011), 154, and NL-35-D (Larson and Schultz 2008, Siedek et al 2011) vaccine strains; all of which are type 2 strains, attenuated in cell culture, and inoculated using a similar route of administration. It is important to highlight that the last two abovementioned vaccine strains, despite being administered with the same biphasic scheme, required a higher viral titer $\left(10^{7.0} \mathrm{TCID}_{50} / \mathrm{mL}\right)$ than the one used in this study $\left(10^{6.0} \mathrm{TCID}_{50} / \mathrm{mL}\right)$ to achieve an effective protective status (Larson et al 2008, Siedek et al 2011).

The death of $50 \%$ of the unvaccinated animals challenged confirm the virulence of the Cornell-780916 strain. This value also corresponded with that reported in the international literature for this type of experimental group (Hernández et al 2015, Khatri et al 2017). Although there are some variations in the appearance and duration of the clinical signs of CPV, the results of this study were similar to those reported in previously published studies. The onset of disease on day 4 after the challenge is in line with the results described by other authors (Spibey et al 2008, Wilson et al 2013, Khatri et al 2017, Mukthar et al 2021) regardless of the challenge strain used, whereas the appearance of fever and mucoid or haemorrhagic diarrhea occurred at similar moments as those described historically by Meunier et al 1985, during experimental infections.

These results, together with the absence of the CPL0015 strain virulence reversal (data not presented), leave open the possibility of continuing with the development of the cuban vaccine formulation. However, there is still a long way to go, the duration of immunity and stability of the formulation should be explored immediately; as well as the optimization of the vaccination scheme (biphasic and triphasic) against the interference of antiparvovirus antibodies of maternal origin, among other aspects. The perception of the international use of the future cuban vaccine requires evaluating its behaviour against pathogenic antigenic variants: CPV-2a, CPV-2b, and CPV-2c (nonhomologous strains).

Based on the experimental model used and the described experimental conditions, it cis concluded that the cuban isolate, CPL-0015, proved to be safe and induced effective protection against homologous virulent strains of canine parvovirus.

\section{REFERENCES}

Altman KD, Kelman M, Ward MP. 2017. Are vaccine strain, type or administration protocol risk factors for canine parvovirus vaccine failure? Vet Microbiol 210, 8-16.

Aponte FA, Gómez RV, López YM. 2020. Factores predisponentes a la parvovirosis canina registrados en un hospital de Cuautitlan, México. Rev Vet 31, 42-45.

Cunegündes GM, Gomes AL, Viana BF, Paes LH, Shimoya Aldo, et al. 2008. Production and use of egg-yolk antibody for detection of canine parvovirus in feces. Rev Cin Méd Biol 7, 241-248.

Day M. 2008. Vaccine side effects: Fact and fiction. Vet Microbiol 117, 51-58.

De Cramer KG, Stylianides E, Van Vuuren M. 2011. Efficacy of vaccination at 4 and 6 weeks in the control of parvovirus canine. Vet Microbiol 149, 126-132.

Decaro N, Buonavoglia C, Barrs VR. 2020. Canine parvovirus vaccination and immunisation failures: Are we far from disease eradication? Vet Microbiol 247, 108760.

Díaz C, Correa J, Vera V. 2008. Aspectos moleculares del virus de la parvovirosis canina y sus implicaciones en la enfermedad. Rev Med Vet 15, 57-65.

Domínguez OA, Polanco R, Cossío G, Morejón Y, Riquenes Y. 2014. Current trends and perspectives in veterinary vaccine production. Biotec Apl 31, 196-203.

Domínguez OA, Acosta DP, Oliva LM, Rosales BK. 2021. Vacuna cubana contra Pasteurella multocida cunícula: 52 años de inmunización. Rev Salud Anim 43, 1-3.

Dong B, Zhang G, Zhang J, Bai J, Lin W. 2020. A systematic literature review and meta-analysis of characterization of canine parvoviruses 2 prevalent in mainland China. Virol $J 17,1-10$.

Fresneda A, Contreras D, Paneque M, de la Cruz L, Veda C. 2015. Caracterización molecular de un aislamiento cubano de parvovirus canino. VacciMonitor 24, 113-119.

Hao X, He Y, Wang C, Xiao W, Liu R, et al. 2020. The increasing prevalence of CPV-2c in domestic dogs in China. Peer J 8, e9869.

Hernández BB, Catala LF. 2015. Are licensed canine parvovirus (CPV2 and CPV2b) vaccines able to elicit protection against CPV2c subtype in puppies?: a systematic review of controlled clinical trials. Vet Microbiol 180, 1-9.

Khatri R, Poonam MH, Minakshi CS. 2017. Epidemiology, pathogenesis, diagnosis and treatment of canine parvovirus disease in dogs: A mini review abstract. J Vet Sci Med Diagn 6, 2.

Langeveld PJ, Brennan RF, Martínez TJ, Jones DT, Boshuizen SR, et al. 2001. Inactivated recombinant plant virus protects dogs from a lethal challenge with canine parvovirus Vaccine 19, 3661-3670.

Larson LJ, Schultz RD. 2008. Do two current canine parvovirus type 2 and $2 \mathrm{~b}$ vaccines provide protection against the new type $2 \mathrm{c}$ variant. Vet Ther 9, 94-101.

Li G, Ji S, Zhai X, Zhang Y, Liu J, et al. 2017. Evolutionary and genetic analysis of the VP2 gene of canine parvovirus. BMC Genomics 18, 1-13.

Lugo S, Espinosa C, Zamora B, Riera O, Sosa T, et al. 2019. Caracterización microbiológica y genotípica de cepas de Pasteurella multocida asociadas al síndrome respiratorio cunícula. Rev Salud Anim 41, 1-11.

Meunier PC, Cooper BJ, Appel MJ, Lanieu ME, Slauson DO. 1985. Pathogenesis of canine parvovirus enteritis: sequential virus distribution and passive inmunization studies. Vet Pathol 22, 617-624.

Miranda C, Thompson G. 2016. Canine parvovirus: the worldwide occurrence of antigenic variants. J Gen Virol 97, 2043-2057.

Moore G, Hogen E. 2010. Adverse vaccinal events in dogs and cats. Vet Clin Smal Anim 40, 393-407.

Mukthar M, Hasan M. 2021. Update on canine parvovirus infection: a review from the literature. Vet Sc Res Rev 7, 92-100. 
Oliveira HS, Geraldes PF, Lopes RJ, D’Ávila VG, Fischer G, et al. 2010. Exposure of pampas fox (Pseudalopex gymnocercus) and crabeating fox (Cerdocyon thous) from the Southern region of Brazil to canine distemper virus (CDV), canine parvovirus (CPV) and canine coronavirus (CCoV). Braz Arch Biol Technol 53, 593-597.

Peña GI, Vidal FF, Hernández RA. 2020. Factores de riesgo de la parvovirosis canina en cuatro localidades de Cuba. Rev Inv Vet Perú 31, e17824.

Pino RD, Márquez AM, Torres GC, Matos RR, Zamora MY. 2019. Factores de riesgo asociados a la seroprevalencia de parvovirus canino en perros del municipio Boyeros, La Habana, Cuba. Rev Salud Anim 41, 1-6.

Pratelli A, Cavalli A, Martella V, Tempesta M, Decaro N, et al. 2001 Canine parvovirus vaccination: comparison of neutralising antibody responses in pups after inoculation with CPV2 or CPV2b modified live virus vaccines. Clin Diag Lab Immunol 8, 612-615.

Puentes PR. 2012. Parvovirosis canina: situación actual y protección de las vacunas contra las nuevas variantes virales circulantes en la región. Vet 48, 5-10.

Puentes RR, Eliopulos N, Pérez R, Franco G, Sosa K, et al. 2012. Isolation and characterization of canine parvovirus type $2 \mathrm{c}(\mathrm{CPV}-2 \mathrm{c})$ from symptomatic puppies. Braz J Microb 1005-1009.

Qi S, Zhao J, Guo D, Sun D. 2020. A Mini-Review on the epidemiology of canine parvovirus in China. Front Vet Sci 7, 5-11.
Sebastian BP, Felipetto CJ, Kenji ME,Weiblen R, Furtado FE. 2019. New variants of canine parvovirus in dogs in southern Brazil. Arch Virol 164, 1361-1369.

Siedek EM, Schmidt H, Sture GH, Raue R. 2011. Vaccination with canine parvovirus type $2(\mathrm{CPV}-2)$ protects against challenge with virulent CPV-2b and CPV-2c. Berl Munch Tierarztl Wochenschr 124, 58-64.

Spibey N, Greenwood N, Sutton D, Chalmers W, Tarpey I. 2008. Canine parvovirus type 2 protects against virulent challenge with type $2 \mathrm{c}$ virus. Vet Microbiol 128, 48-25.

Voorhees IE, Lee H, Allison AB, Lopez-Astacio R, Goodman LB, et al. 2019. Limited intrahost diversity and background evolution accompany 40 years of canine parvovirus host adaptation and spread. J Virol 94, e01162-19.

Wilson S, Stirling C, Borowski S, Thomas A, King V, et al. 2013. Vaccination of dogs with Duramune DAPPi+LC protects against pathogenic canine parvovirus type $2 \mathrm{c}$ challenge. Vet Rec 172, 662-672.

Ying EY, Peaston A, Woolford L, Khuu SJ, Wallace G, et al. 2020. Diagnostic challenges in canine parvovirus $2 \mathrm{c}$ in vaccine failure cases. Viruses 12, 980-93.

Zhou P, Zeng W, Zhang X, Li S. 2017. The genetic evolution of canine parvovirus-A new perspective. PLOS ONE 12, e0175035.

Zhuang QY, Qiu Y, Pan ZH. 2019. Genome sequence characterization of canine parvoviruses prevalent in the Sichuan province of China. Transbound Emerg Dis 66, 897-907. 